\title{
Androgen deprivation therapy: progress in understanding mechanisms of resistance and optimizing androgen depletion
}

\author{
William P Harris, \\ Medical Oncology Fellow at Fred Hutchinson Cancer Research Center and University of \\ Washington School of Medicine, Seattle, WA, USA
}

Elahe A Mostaghel,

Assistant Member in the Divisions of Human Biology and Clinical Research, Fred Hutchinson Cancer Research Center and Assistant Professor in the Department of Medicine, University of Washington School of Medicine, Seattle, WA, USA

Peter S Nelson, and Member in the Divisions of Human Biology and Clinical Research, Fred Hutchinson Cancer Research Center and Professor in the Department of Medicine, University of Washington School of Medicine, Seattle, WA, USA

\begin{abstract}
Bruce Montgomery ${ }^{*}$
Associate Member in the Divisions of Human Biology and Clinical Research, Fred Hutchinson Cancer Research Center and Associate Professor in the Department of Medicine, University of Washington School of Medicine, Seattle, WA, USA
\end{abstract}

\section{SUMMARY}

Androgen deprivation therapy remains a critical component of treatment for men with advanced prostate cancer, and data supports its use in metastatic disease and in conjunction with surgery or radiation in specific settings. Alternatives to standard androgen deprivation therapy, such as intermittent androgen suppression and estrogen therapy, hold the potential to improve toxicity profiles while maintaining clinical benefit. Current androgen deprivation strategies seem to incompletely suppress androgen levels and androgen-receptor-mediated effects at the tissue level. Advances in the understanding of mechanisms that contribute to castration-resistant prostate cancer are leading to rationally designed therapies targeting androgen metabolism and the androgen receptor. The results of large trials investigating the optimization of primary androgen deprivation therapy, including evaluation of intermittent androgen suppression and estrogen patch assessment, as well as phase III studies of novel androgen synthesis inhibitors, such as abiraterone acetate, are eagerly awaited.

\footnotetext{
*Correspondence Department of Medicine, University of Washington, 1959 Northeast Pacific, Seattle, WA 98195, USA, rbmontgo@u.washington.edu.

Medscape, LLC is pleased to provide online continuing medical education (CME) for this journal article, allowing clinicians the opportunity to earn CME credit. Medscape, LLC is accredited by the Accreditation Council for Continuing Medical Education (ACCME) to provide CME for physicians. Medscape, LLC designates this educational activity for a maximum of 1.0 AMA PRA Category 1 Credits $^{\text {TM }}$. Physicians should only claim credit commensurate with the extent of their participation in the activity. All other clinicians completing this activity will be issued a certificate of participation. To receive credit, please go to http://www.medscape.com/ $/ \mathrm{cme} / \mathrm{hcp}$ and complete the post-test.

Competing interests

PS Nelson has declared associations with the following company/organization: GlaxoSmithKline. B Montgomery has declared an association with the following company/organization: Cougar Biotechnology. See the article online for full details of the relationships. WP Harris and EA Mostaghel declared no competing interests.
} 


\section{Keywords}

androgen; cancer; mechanism; prostate; resistance

\section{INTRODUCTION}

Prostate cancer is the most common solid tumor and the second most common cause of cancer death among men in the United States, ${ }^{1}$ and its worldwide incidence is the second highest of all male non-skin malignancies. ${ }^{2}$ At present, approximately $85 \%$ of newly diagnosed prostate cancers are localized to the prostate, with the remainder representing invasive or metastatic disease. ${ }^{3}$ Recognized treatment options for early-stage disease include surgery, radiation therapy and active surveillance. For many men with metastatic or high-risk localized disease, androgen deprivation is a critical component of therapy, either alone or in combination with other modalities. This Review will provide an overview of historical and contemporary approaches to androgen deprivation in patients with advanced prostate cancer, and address its current clinical indications: either as monotherapy or in combination with local therapy. Mechanisms of resistance to classical androgen deprivation, which might be exploited to therapeutic effect in early and late-stage cancer, will be discussed, as will several promising new therapies that are undergoing early assessment.

\section{HISTORICAL APPROACHES TO ANDROGEN DEPRIVATION THERAPY}

Prostate cancer has been recognized as an androgen-sensitive disease since the seminal work of Huggins and Hodges in 1941.4 Nearly seven decades later, clinicians and researchers still struggle to completely understand and optimize androgen deprivation therapy (ADT). Testosterone and dihydrotestosterone (DHT) are the two major androgens in men, with testosterone present mainly in circulation, and DHT the primary androgen in prostatic tissues. The Leydig cells of the testis produce $90-95 \%$ of circulating testosterone, with 5-10\% of circulating testosterone synthesized in the adrenal glands. ${ }^{5}$ In contrast, DHT primarily arises from conversion of testosterone to DHT at the tissue level by the two isoforms of 5[alpha]reductase, S5A1 and S5A2. About 25\% of circulating DHT is produced in the testes, with the remaining $75 \%$ produced in tissues such as the prostate and skin.6 The standard paradigm describes circulating testosterone and other androgen precursors diffusing through the prostate, with uptake and transport into the prostatic epithelium, and subsequent tissue-level conversion to DHT.

Androgens function predominantly through their action on the androgen receptor (AR), a member of the steroid hormone receptor family of ligand-activated nuclear transcription factors. Compared with testosterone, DHT binds the AR in a more stable manner, leading to a 10 -fold increase in transcriptional activation, which makes DHT the primary ligand and effector of AR-mediated signaling at the tissue level. ${ }^{6}, 7$ The AR exists in the cytoplasm, bound to heat shock proteins, which stabilize the AR and allow androgen binding. Upon ligand interaction, the AR homodimerizes, undergoing phosphorylation and translocation to the nucleus, where it binds androgen response elements and induces transcription of target genes involved in cell-cycle regulation and proliferation (Figure 1). $8^{-10}$ As androgens have a critical role in driving prostate cancer growth, physicians have utilized ADT to achieve drastic reductions in the rates of testicular androgen synthesis and levels of circulating androgens, thereby minimizing AR ligand availability and subsequent AR-mediated proliferative effects on the prostate. Common methods of ADT include orchiectomy or medical castration through the chronic administration of gonadotropin-releasing hormone $(\mathrm{GnRH})$ agonists; other medical approaches include estrogen therapy, which also results in impaired androgen production and castrate levels of circulating testosterone. 
Historically, circulating testosterone levels have been used to assess the efficacy of androgen depletion, with a target total testosterone level below 50|ng/dl $(<1.74 \mid \mathrm{nmol} / \mathrm{l})$. This target is defined on the basis of the level of suppression achieved with surgical castration, and remains the benchmark for evaluating the efficacy of agents such as GnRH agonists.11 In men with prostate cancer, orchiectomy reduces serum testosterone to anorchid levels within $12 \mid \mathrm{h}$, with rapid reduction of tumor burden, glandular atrophy, and involution of the prostate. In xenograft models, cellular proliferation decreases with castration, and apoptotic rates increase within $3^{\wedge}$ days. Interestingly, proliferation and apoptosis indices in both malignant and benign tissue normalize by day 7-10 after castration, suggesting limited extent and duration of cell death with ADT.12 The use of GnRH agonists (with antiandrogens) induces similar but delayed effects, as a result of the slower decline in testosterone levels seen with these agents compared with surgical castration.13,14 Additionally, castration induces epithelial cell-cycle arrest of malignant cells in G0-G1 phase. Given that these cells eventually exit cell-cycle arrest and progress to androgen independence, concurrent targeting of the arrested cell population might represent a potential therapeutic strategy to prevent progression to castration-resistant prostate cancer (CRPC). 15

In current clinical practice, the use of GnRH agonists is the preferred means of achieving anorchid testosterone levels. Cyclic GnRH stimulation of the anterior pituitary stimulates the release of luteinizing hormone (LH), which in turn stimulates testicular androgen synthesis in the normal host. Depot GnRH agonists, such as leuprolide, goserelin, buserelin and others, induce an initial transient increase in LH release, followed by tachyphylaxis resulting from nonphysiologic and nonpulsatile stimulation of GnRH receptors. Administration of high levels of other sex steroids, such as progesterone or estrogens, achieves similar suppression of LH release through feedback inhibition of the hypothalamic-pituitary axis.

The potential therapeutic benefit of inhibiting both AR ligand production and binding led to the development of AR antagonists, such as bicalutamide, flutamide and nilutamide; however, the relative benefit of combining GnRH agonists or orchiectomy with AR antagonists remains an area of controversy. Meta-analyses of randomized studies have suggested a small, albeit consistent, benefit to the use of combined blockade in the treatment of men with metastatic disease. ${ }^{16}$ Some consensus panels recommend consideration of combined androgen blockade, given an apparent $5 \%$ survival advantage and limited additional toxicity over castration alone. ${ }^{11}$ At present, combined blockade is most commonly employed as a component of neoadjuvant and concurrent ADT with radiation therapy, and in intermittent androgen suppression. ${ }^{17,18}$

\section{INDICATIONS FOR ANDROGEN DEPRIVATION THERAPY}

ADT prolongs overall survival, produces an objective response in bone and soft tissue metastatic disease, relieves bone pain, and suppresses PSA levels in 80-90\% of patients with metastatic prostate cancer. While a dramatic initial response to therapy is common, ADT in metastatic disease is considered palliative, as disease progression despite anorchid serum testosterone occurs at a median of $2-3^{\wedge}$ years, with a subsequent expected survival of $16-$ $18^{\wedge}$ months from the time of progression. ${ }^{19}$ Duration of response to therapy varies, with 5-10\% of patients remaining alive $10^{\wedge}$ years after initiating ADT. ${ }^{20} \mathrm{~A}$ standard approach for patients with metastatic prostate cancer has been the use of either a GnRH agonist or orchiectomy as monotherapy. ${ }^{16}$ For patients with symptomatic metastatic disease, a nonsteroidal antiandrogen is often used before or at initiation of GnRH agonists to prevent adverse effects from the resultant initial LH and testosterone surge. In asymptomatic patients with metastatic disease, controversy exists over whether to initiate ADT immediately or to defer therapy. Although immediate initiation of ADT does not confer a clear overall survival advantage in these patients, it does improve cancer-specific survival and reduces the incidence of spinal cord compression, pathologic fracture and the need for palliative transurethral resection of the prostate. ${ }^{21}$ 
Numerous randomized trials have demonstrated a benefit of combining androgen deprivation with definitive radiation therapy in men with locally advanced or high-grade disease (Table 1). ${ }^{22-25}$ Potential benefits of androgen deprivation include optimized radiation-induced apoptosis in local disease, and suppression of systemic micrometastasis. ${ }^{26}$ The trials reported in Table 1 varied with respect to the definition of high-risk disease, as well as the timing and duration of ADT, but essentially all have shown significant improvements in long-term local, distant and biochemical control. Although improvements in overall survival have been less convincingly demonstrated, the combination of ADT and radiation therapy is widely accepted as improving outcomes for patients with locally advanced disease. Most regimens include ADT monotherapy or combined blockade for 2 or $3^{\wedge}$ months before radiation therapy, concurrent therapy, and a variable duration of adjuvant treatment. Retrospective analyses suggest a benefit of combined blockade, ${ }^{27}$ although the relative benefits of combined blockade versus monotherapy in this setting have not been evaluated in randomized trials. For patients with intermediate-risk disease without significant comorbidity, $6^{\wedge}$ months of combined blockade plus radiation therapy carries an overall survival advantage compared with radiation therapy alone. ${ }^{17,25}$ One, as yet unpublished, randomized study in patients with high-risk cancer have described better outcomes with $3^{\wedge}$ years' ADT compared with $6^{\wedge}$ months' ADT. ${ }^{28}$ The benefits of combining ADT with radiation therapy have not been compared to those of dose escalation for patients with intermediate-risk disease. Until such studies have been completed, either $6^{\wedge}$ months of ADT or increasing the radiation dose remain standard options for patients with intermediate-risk disease undergoing radiation therapy.

Adjuvant ADT has been clearly shown to improve overall survival in men found to have nodal metastasis after prostatectomy. 29,30 In a randomized, multi-institutional trial that assessed men with nodal disease detected at radical prostatectomy with pelvic lymphadenectomy, immediate GnRH agonist therapy or bilateral orchiectomy was associated with improved overall survival, cancer-specific survival and progression-free survival, compared with deferred hormonal therapy. After an 11.9-year median follow-up, median survival duration differed significantly between the immediate and deferred therapy subgroups (13.9^years vs $11.3^{\wedge}$ years). This finding is in contrast to that of the EORTC trial 30846, which found no survival advantage of immediate ADT in patients in whom prostatectomy was not completed. ${ }^{31}$ Messing et al.30 attribute this discrepancy to the large volume of residual disease in EORTC trial participants, hypothesizing that the benefits of ADT are probably greatest in patients with a low volume of residual malignant tissue.

Additional aspects of ADT that remain controversial include the timing of treatment in patients with biochemical relapse, the utility of ADT alongside salvage radiotherapy, and the benefits of ADT alone in patients with locally advanced prostate cancer. ${ }^{32,33}$ Contemporary analyses suggest that the use of primary ADT does not provide significant improvements in survival for the majority of elderly men with localized disease. ${ }^{34}$

\section{TOXICITIES OF ANDROGEN DEPRIVATION THERAPY}

Several Reviews have described the toxicities of androgen deprivation. ${ }^{35,36}$ ADT is associated with a number of adverse effects on quality of life, including sexual dysfunction, muscle atrophy, osteoporosis, hot flashes, fatigue, gynecomastia, anemia and, in some patients, depression and cognitive dysfunction. ${ }^{37}$ ADT can also induce the metabolic syndrome, which seems to account for the increased incidences of diabetes and cardiovascular morbidity and mortality associated with ADT. ${ }^{35,36}$

\section{MECHANISMS OF RESISTANCE TO ANDROGEN DEPRIVATION THERAPY}

The efficacy of different approaches to androgen suppression has been defined according to the capability to consistently achieve serum levels of total testosterone below 50|ng/dl. When 
progression occurs despite maintenance of anorchid serum androgen levels, the disease has been considered to be 'androgen-independent' or 'hormone-refractory'. These labels result from the presumption that serum androgen concentrations reflect androgen levels in the prostate and metastatic sites; nowadays, the term 'castration-resistant' is preferred. Several theories addressing the mechanism by which CRPC develops have been well described in the literature (Figure 2) ${ }^{9}{ }^{9}{ }^{19}$ In general, these theories either incorporate the concept of continued AR signaling via alternative pathways, or invoke truly AR-independent mechanisms.

Continued signaling through the AR has been postulated to occur via AR amplification, or via AR mutation that increases sensitivity to DHT and nonandrogenic steroid molecules or antiandrogens. ${ }^{9,38}$ Other possible AR-dependent mechanisms include activation of the AR or downstream effectors via ligand-independent modifications, such as AR phosphorylation; 39 cross-talk with activated tyrosine kinase receptors, such as EGFR; or a change in the balance of coactivators and corepressors. Most proposed mechanisms implicate increased sensitivity of the AR to low-level androgens, consistent with the finding that, for wild-type AR, the ligand binding domain is necessary for the development of resistance to castration. ${ }^{38}$ Other hypotheses suggest that resistance develops via bypass of intact AR pathways, and protection of cells from castration-induced apoptosis through androgen-independent upregulation of antiapoptotic molecules, such as Bcl-2. ${ }^{40}$ Finally, some authors have suggested that AR-negative stem cells that are resistant to castration might continuously repopulate the prostate with both androgendependant and androgen-independent cells. ${ }^{19}$

\section{PERSISTENCE OF TISSUE ANDROGENS DESPITE CASTRATION}

Evidence suggests that patients with anorchid serum testosterone concentrations maintain prostatic androgen levels sufficient to support AR signaling and cancer cell survival. Early reports demonstrated that, in patients with localized prostate cancer, orchiectomy or medical castration suppressed intraprostatic DHT levels by only $75-80 \%$, leaving tissue DHT levels well within a range anticipated to activate the AR.41 More-recent analysis of tissue from men undergoing short-term ADT demonstrated that prostatic testosterone and DHT levels were reduced by $70-80 \%$, although very limited suppression of transcripts for androgen-regulated genes was observed.42 In patients with established CRPC, prostate tumors contained testosterone levels equivalent to those found in the prostatic tissue of untreated men, with DHT levels decreased to $20 \%$ of those in untreated tissue. 43 Soft tissue metastases in patients with anorchid serum testosterone contain levels of testosterone that are up to three times higher than those in prostate tumors in eugonadal men. ${ }^{44}$ Transcript levels of enzymes involved in androgen synthesis were upregulated in the same tumors (8-30-fold), suggesting that tumoral synthesis of androgens from cholesterol might occur. Bone metastases in patients with CRPC also contain intact enzymatic pathways for the conversion of adrenal androgens to DHT. ${ }^{45}$ Finally, androgen-independent prostate cancer cell lines synthesize testosterone from radiolabeled cholesterol in vitro ${ }^{, 46}$ and human prostate cancer xenografts are capable of synthesizing DHT from acetate and cholesterol, confirming that tumoral androgen synthesis is possible. ${ }^{47}$ Thus, experimental and clinical evidence suggests that enzymes of tumoral steroid metabolism can support CRPC, and might provide multiple new targets for therapeutic intervention.

\section{CONTEMPORARY APPROACHES TO ANDROGEN DEPRIVATION THERAPY}

Alternatives to the standard ADT discussed above have been explored, with the hope of reducing toxicity and circumventing various mechanisms of resistance to therapy. Compelling preclinical data have suggested that allowing testosterone recovery after a period of androgen deprivation would help prevent the development of resistance by lessening the selection pressure for tumor growth in low-androgen conditions. ${ }^{48}$ Early institutional series of 
intermittent androgen suppression (IAS) demonstrated that 'cycling' - periods of androgen deprivation followed by off-cycle periods - was safe, and provided similar, if not superior, rates of control, and improved cognitive, sexual and bone health, compared with monotherapy. 49-51 The design of IAS studies has varied substantially; for example, employing GnRH agonists with or without a nonsteroidal antiandrogen, on-treatment cycles lasting $3-9^{\wedge}$ months, and different triggers for initiation of subsequent cycles.49 Several trials, including various phase II studies and interim analyses of phase III trials, suggest improvement in terms of quality of life, cardiovascular events and osteoporosis, among other benefits, with IAS.52,53 Rates of overall survival and progression to CRPC seem to be equivalent to those seen with continuous therapy, according to several phase II trials and early phase III data. ${ }^{18}$ In preliminary results from one phase III trial of 201 patients with biochemical progression who were randomized to IAS versus combined androgen blockade, no significant differences were noted between groups in time to progression to CRPC at a median follow-up of $31^{\wedge}$ months. ${ }^{54}$ Initial results from a second phase III trial of 766 patients with locally advanced or metastatic prostate cancer who were randomized to IAS or combined androgen blockade showed no difference in overall survival or progression to CRPC at a median follow-up of $51^{\wedge}$ months. ${ }^{55}$ As patients receive IAS drugs at approximately half the rate that they would with continuous treatment, this approach might reduce costs, with equivalent control rates. The final results of several ongoing large phase III trials are eagerly awaited.

Estrogens have had something of a renaissance as an alternative and viable mode of androgen deprivation in men with prostate cancer. Estrogens can achieve anorchid testosterone levels through negative feedback of the hypothalamic-pituitary axis, thus reducing $\mathrm{LH}$ production, 56 and might engage multiple additional mechanisms to suppress prostate cancer growth. First, estrogen activates both the [alpha] and [beta] isoforms of estrogen receptor (ER). ER[alpha] induces proliferation, and is necessary for prostate carcinogenesis in multiple models.57 ER [beta], however, seems to suppress carcinogenesis and slow prostate cancer growth.58 ER [beta] activation is, therefore, another potential mechanism of suppressing prostate cancer growth. Estrogens might also suppress tumor growth through competition with androgens for AR binding, and reduce testicular testosterone production.59 In patients with CRPC, the nonsteroidal estrogen diethylstilbestrol (DES) reduces circulating levels of adrenal androgens, 60 potentially abrogating a source of androgens for tissue metabolism to testosterone and DHT.

DES has long been used to treat advanced prostate cancer, with demonstrated efficacy as a primary hormonal agent and, more recently, as a secondary hormonal manipulation. In the Veterans Administration Cooperative Urological Research Group (VACURG) studies, DES at higher doses (5 $\mid \mathrm{mg}$ daily) delayed progression to metastatic disease and improved cancerspecific survival in patients with locally advanced disease compared with orchiectomy; however, overall survival was similar for the two treatments, owing to high rates of fatal cardiovascular toxicity. ${ }^{61}$ In CRPC, low-dose DES produces PSA responses, lasting an average of $6^{\wedge}$ months, in $30-40 \%$ of patients, with symptom improvement noted in at least one trial. These benefits are obtained at the expense of increased cardiovascular toxicity, occurring in $5-28 \%$ of cases.56

Renewed interest in estrogen-based therapy has been driven by the recognition that estrogens might offset some of the toxicity of standard ADT. Transdermal administration of estrogen avoids hepatic first-pass metabolism, limiting the risk of cardiovascular toxicity. In small institutional series, the potential advantages of transdermal estradiol over standard ADT were found to include improvements in bone density, dyslipidemia and some elements of cognitive function in men with advanced prostate cancer.62,63 The majority of the benefits of estradiol have been shown in patients receiving this agent as primary therapy, and its utility in men with CRPC is limited, as it is associated with relatively low PSA response rates.64, ${ }^{65}$ Larger studies 
investigating the use of transdermal estrogens and ER[beta]-specific agonists might provide more insights into how estrogen therapy could be best incorporated into standard ADT.

\section{NEW HORIZONS IN ANDROGEN DEPRIVATION THERAPY}

As discussed above, an incomplete response to ADT and subsequent disease progression might be mediated by continued AR signaling. Several investigational drugs in preclinical and early clinical trials have been designed to address this potential mechanism of resistance.

MDV3100 (Mediviation Inc., San Francisco, CA) is a small-molecule, pure AR antagonist that inhibits AR nuclear translocation and DNA binding. The drug was selected as a result of its activity in models of AR overexpression, in which conventional antiandrogens develop agonist properties. Xenograft models have demonstrated significant reductions in cancer volume with MDV3100 in comparison with bicalutamide monotherapy, which showed no effect. ${ }^{66}$ Recently presented results of phase I-II studies in humans showed significant PSA reductions in 13 of 14 patients with CRPC who were treated for over $4^{\wedge}$ weeks with MDV3100. The drug was well tolerated, and no clinical or radiographic progression was noted in the six patients who were observed for $14^{\wedge}$ weeks,. 67

Abiraterone acetate (Cougar Biotechnology, Los Angeles, CA) is an orally administered, specific inhibitor of CYP17A1, a rate-limiting enzyme in androgen biosynthesis. Inhibition of enzymatic activity at two sequential steps in the androgen biosynthesis pathway leads to reduced dihydroepiandrosterone and androstenedione synthesis, which limits subsequent conversion to testosterone and DHT. Given the key role of CYP17A1 in androgen production, inhibition of this enzyme would be expected to suppress androgen production in all endocrine organs, including the testis, adrenal glands, and postulated tumoral sites of androgen production. ${ }^{68}$ Abiraterone acetate has been evaluated in a phase I trial of patients with metastatic CRPC who had undergone multiple hormonal manipulations before enrollment. 58 Significant clinical activity was noted in this trial, with PSA reductions of at least 50\% occurring in 57\% of patients, and symptomatic and radiographic improvement documented in some cases. Preliminary phase II data of abiraterone acetate in patients with CRPC after failure of docetaxel-based chemotherapy have also been presented. Overall, the treatment was well tolerated, with $45 \%$ of patients achieving a PSA nadir to less than $50 \%$ of baseline level, and $38 \%$ of patients demonstrating a partial radiologic response or stable disease at $12^{\wedge}$ weeks. 69 The relatively high response rates to the CYP17A1 inhibitor abiraterone acetate and the AR antagonist MDV3100 in patients with CRPC supports the hypothesis that tumoral androgen synthesis and continued AR signaling have a role in the pathogenesis of CRPC. 68,70

Other agents are being developed to suppress tissue androgen production. An exciting drug in preclinical evaluation, VN-124-1 (Tokai Pharmaceuticals, Inc., Cambridge, MA), combines the advantages of CYP17A1 inhibition, AR antagonism and reduced AR protein synthesis. In vitro models have demonstrated potent $\mathrm{AR}$ antagonism in binding assays and inhibition of CYP17A1 enzymatic activity, while in vivo xenograft models showed reduced tumor burden in mice treated with $\mathrm{VN}-124-1$ versus those undergoing castration or bicalutamide monotherapy. ${ }^{71}$ Suppression of AR protein synthesis-an additional mechanism of action that might prevent resistance developing through AR amplification - was also demonstrated both in vitro and in vivo. This combination of effects might also suppress AR ligand activity of upstream steroid precursors, which theoretically accumulate with CYP17A1 inhibition, and which might bind the AR and initiate downstream effects. ${ }^{71,72}$

New combinations of agents that are already commercially available might also improve androgen deprivation strategies. An ongoing study ${ }^{73}$ assessing men with CRPC and biochemical progression combines dutasteride and antiandrogen therapy, hypothesizing that 
the increase in the ratio of testosterone|:|DHT provided by dutasteride might improve the efficacy of antiandrogens.

\section{CONCLUSIONS}

Advances in our understanding of the mechanisms contributing to CRPC are leading to rationally designed therapies that target androgen metabolism and the AR. Minimizing the adverse effects of therapy while diminishing androgen-mediated activity at the tissue level through inhibition of testicular, adrenal and tumoral androgen production holds promise for improving survival outcomes. The results of large trials investigating the optimization of primary ADT, including the evaluation of IAS and estrogen patches, as well as phase III studies of novel androgen synthesis inhibitors, such as abiraterone acetate, are eagerly awaited. The use of novel agents, either as monotherapy or in combination, might prevent the development and progression of CRPC, improve quality of life, and extend survival.

\section{REVIEW CRITERIA}

Information sources used in this Review were obtained through searching the PubMed and MEDLINE databases between the years 1998 and 2008, with references limited to those published in English. Search parameters, used alone or in combination, included "prostate cancer", "castration", "mechanism", "resistance", "androgen", "estrogen", "DES", "intermittent", "GnRH", "androgen deprivation therapy", "radiation", "VN124", "CYP17" and "radiosensitization". Additional articles and abstracts were identified from the reference lists of articles obtained from the initial search and from the abstract database of the American Society of Clinical Oncology, searched between 2005 and 2008 for selected topics.

\section{KEY POINTS}

- Androgen deprivation therapy remains a critical component of treatment in men with advanced prostate cancer, and data supports its use in metastatic disease and in conjunction with surgery or radiation therapy in specific settings

- Alternatives to standard androgen deprivation therapy, such as intermittent androgen suppression and estrogen therapy, hold the potential to improve toxicity profiles while maintaining clinical benefit

- Current androgen deprivation strategies seem to incompletely suppress androgen levels and AR-mediated effects at the tissue level

- Multiple mechanisms are implicated in the development of castration resistance, but the majority involve continued activation of the AR, which might be targeted by novel therapeutic agents

\section{Acknowledgments}

This work was made possible by funding through the NIH/NCI Pacific Northwest Prostate Cancer SPORE grant P50CA97186 (to PS Nelson and B Montgomery) and the Kirschstein-NRSA T32 training grant (to WP Harris).

\section{References}

1. Jemal A, et al. Cancer statistics, 2008. CA Cancer J Clin 2008;58:71-96. [PubMed: 18287387] 
2. Kamangar F, et al. Patterns of cancer incidence, mortality, and prevalence across five continents: defining priorities to reduce cancer disparities in different geographic regions of the world. J Clin Oncol 2006;24:2137-2150. [PubMed: 16682732]

3. Cooperberg MR, et al. The contemporary management of prostate cancer in the United States: lessons from the Cancer of the Prostate Strategic Urologic Research Endeavor (CAPSURE), a national disease registry. J Urol 2004;171:1393-1401. [PubMed: 15017184]

4. Huggins C, Hodges CV. Studies on prostate cancer, I: the effect of castration, of estrogen and of androgen injection on serum phosphatases in metastatic carcinoma of the prostate. Cancer Res 1941;1:293-297.

5. Labrie FMD. Adrenal androgens and intracrinology. Semin Reprod Med 2004;22:299-309. [PubMed: 15635498]

6. Takashi I, et al. The role of testosterone in the pathogenesis of prostate cancer. Int J Urol 2008;15:472480. [PubMed: 18430151]

7. Askew EB, et al. Modulation of androgen receptor activation function 2 by testosterone and dihydrotestosterone. J Biol Chem 2007;282:25801-25816. [PubMed: 17591767]

8. Feldman BJ, Feldman D. The development of androgen-independent prostate cancer. Nat Rev Cancer 2001;1:34-45. [PubMed: 11900250]

9. Taplin ME. Drug Insight: role of the androgen receptor in the development and progression of prostate cancer. Nat Clin Pract Oncol 2007;4:236-244. [PubMed: 17392714]

10. Mostaghel EA, et al. Intraprostatic androgens and androgen-regulated gene expression persist after testosterone suppression: therapeutic implications for castration-resistant prostate cancer. Cancer Res 2007;67:5033-5041. [PubMed: 17510436]

11. Loblaw DA, et al. Initial hormonal management of androgen-sensitive metastatic, recurrent, or progressive prostate cancer: 2007 update of an American Society of Clinical Oncology practice guideline. J Clin Oncol 2007;25:1596-1605. [PubMed: 17404365]

12. Ohlson N, et al. Cell proliferation and apoptosis in prostate tumors and adjacent non-malignant prostate tissue in patients at different time-points after castration treatment. Prostate 2005;62:307315. [PubMed: 15389788]

13. Mercader M, et al. Early effects of pharmacological androgen deprivation in human prostate cancer. BJU Int 2007;99:60-67. [PubMed: 17227493]

14. Montironi R, et al. Effects of combination endocrine treatment on normal prostate, prostatic intraepithelial neoplasia, and prostatic adenocarcinoma. J Clin Pathol 1994;47:906-913. [PubMed: 7525657]

15. Agus DB, et al. Prostate cancer cell cycle regulators: response to androgen withdrawal and development of androgen independence. J Natl Cancer Inst 1999;91:1869-1876. [PubMed: 10547394]

16. Maximum androgen blockade in advanced prostate cancer: an overview of the randomised trials. Prostate Cancer Trialists' Collaborative Group. Lancet 2000;355:1491-1498. [No authors listed]. [PubMed: 10801170]

17. D'Amico AV, et al. 6-Month androgen suppression plus radiation therapy vs radiation therapy alone for patients with clinically localized prostate cancer: a randomized controlled trial. JAMA 2004;292:821-827. [PubMed: 15315996]

18. Boccon-Gibod L, et al. The role of intermittent androgen deprivation in prostate cancer. BJU Int 2007;100:738-743. [PubMed: 17662079]

19. Pienta KJ, Bradley D. Mechanisms underlying the development of androgen-independent prostate cancer. Clin Cancer Res 2006;12:1665-1671. [PubMed: 16551847]

20. Tangen CM, et al. Ten-year survival in patients with metastatic prostate cancer. Clin Prostate Cancer 2003;2:41-45. [PubMed: 15046683]

21. Immediate versus deferred treatment for advanced prostatic cancer: initial results of the Medical Research Council Trial. The Medical Research Council Prostate Cancer Working Party Investigators Group. Br J Urol 1997;79:235-246. [No authors listed]. [PubMed: 9052476]

22. Bolla M, et al. Long-term results with immediate androgen suppression and external irradiation in patients with locally advanced prostate cancer (an EORTC study): a phase III randomised trial. Lancet 2002;360:103-108. [PubMed: 12126818] 
23. Lawton CA, et al. Updated results of the phase III Radiation Therapy Oncology Group (RTOG) trial 85-31 evaluating the potential benefit of androgen suppression following standard radiation therapy for unfavorable prognosis carcinoma of the prostate. Int J Radiat Oncol Biol Phys 2001;49:937-946. [PubMed: 11240234]

24. Pilepich MV, et al. Phase III radiation therapy oncology group (RTOG) trial 86-10 of androgen deprivation adjuvant to definitive radiotherapy in locally advanced carcinoma of the prostate. Int $\mathrm{J}$ Radiat Oncol Biol Phys 2001;50:1243-1252. [PubMed: 11483335]

25. Denham JW, et al. Short-term androgen deprivation and radiotherapy for locally advanced prostate cancer: results from the Trans-Tasman Radiation Oncology Group 96.01 randomised controlled trial. Lancet Oncol 2005;6:841-850. [PubMed: 16257791]

26. Pollack A, et al. Lack of prostate cancer radiosensitization by androgen deprivation. Int J Radiat Oncol Biol Phys 2001;51:1002-1007. [PubMed: 11704324]

27. D'Amico AV, et al. Risk of prostate cancer recurrence in men treated with radiation alone or in conjunction with combined or less than combined androgen suppression therapy. J Clin Oncol 2008;26:2979-2983. [PubMed: 18565884]

28. Bolla M, et al. Concomitant and adjuvant androgen deprivation (ADT) with external beam irradiation (RT) for locally advanced prostate cancer: $6^{\wedge}$ months versus $3^{\wedge}$ years ADT—results of the randomized EORTC Phase III trial 22961. JCO 2007 Annual Meeting Proceedings Part 1 25, 5014. J Clin Oncol 2007;25(18 Suppl):5014. [abstract \#5014].

29. Messing EM, et al. Immediate hormonal therapy compared with observation after radical prostatectomy and pelvic lymphadenectomy in men with node-positive prostate cancer. N Engl J Med 1999;341:1781-1788. [PubMed: 10588962]

30. Messing EM, et al. Immediate versus deferred androgen deprivation treatment in patients with nodepositive prostate cancer after radical prostatectomy and pelvic lymphadenectomy. Lancet Oncol 2006;7:472-479. [PubMed: 16750497]

31. Schröder FH, et al. Early versus delayed endocrine treatment of pN1-3 M0 prostate cancer without local treatment of the primary tumor: results of the European Organization for the Research and Treatment of Cancer 30846-a phase III study. J Urol 2004;172:923-927. [PubMed: 15310999]

32. Stephenson AJ, et al. Salvage radiotherapy for recurrent prostate cancer after radical prostatectomy. JAMA 2004;291:1325-1332. [PubMed: 15026399]

33. Freedland SJ, Moul JW. Prostate specific antigen recurrence after definitive therapy. J Urol 2007;177:1985-1991. [PubMed: 17509277]

34. Lu-Yao GL, et al. Survival following primary androgen deprivation therapy among men with localized prostate cancer. JAMA 2008;300:173-181. [PubMed: 18612114]

35. Harle LK, et al. Endocrine complications of androgen-deprivation therapy in men with prostate cancer. Clin Adv Hematol Oncol 2006;4:687-696. [PubMed: 17099626]

36. Michaelson MD, et al. Management of complications of prostate cancer treatment. CA Cancer J Clin 2008;58:196-213. [PubMed: 18502900]

37. Seruga B, Tannock IF. Intermittent androgen blockade should be regarded as standard therapy in prostate cancer. Nat Clin Pract Oncol 2004;5:574-576. [PubMed: 18628739]

38. Chen CD, et al. Molecular determinants of resistance to antiandrogen therapy. Nat Med 2004;10:3339. [PubMed: 14702632]

39. Kraus S, et al. Receptor for activated C kinase 1 (RACK1) and Src regulate the tyrosine phosphorylation and function of the androgen receptor. Cancer Res 2006;66:11047-11054. [PubMed: 17108144]

40. Setlur SR, Rubin MA. Current thoughts on the role of the androgen receptor and prostate cancer progression. Adv Anat Pathol 2005;12:265-270. [PubMed: 16210922]

41. Liu J, et al. Acute effects of testicular and adrenal cortical blockade on protein synthesis and dihydrotestosterone content of human prostate tissue. J Clin Endocrinol Metab 1985;61:129-133. [PubMed: 2581988]

42. Mostaghel EA, et al. Intraprostatic androgens and androgen-regulated gene expression persist after testosterone suppression: therapeutic implications for castration-resistant prostate cancer. Cancer Res 2007;67:5033-5041. [PubMed: 17510436] 
43. Mohler JL, et al. The androgen axis in recurrent prostate cancer. Clin Cancer Res 2004;10:440-448. [PubMed: 14760063]

44. Montgomery RB, et al. Maintenance of intratumoral androgens in metastatic prostate cancer: a mechanism for castration-resistant tumor growth. Cancer Res 2008;68:4447-4454. [PubMed: 18519708]

45. Stanbrough M, et al. Increased expression of genes converting adrenal androgens to testosterone in androgen-independent prostate cancer. Cancer Res 2006;66:2815-2825. [PubMed: 16510604]

46. Dillard PR, et al. Androgen-independent prostate cancer cells acquire the complete steroidogenic potential of synthesizing testosterone from cholesterol. Mol Cell Endocrinol 2008;295:115-120. [PubMed: 18782595]

47. Locke JA, et al. Androgen levels increase by intratumoral de novo steroidogenesis during progression of castration-resistant prostate cancer. Cancer Res 2008;68:6407-6415. [PubMed: 18676866]

48. Sato N, et al. Intermittent androgen suppression delays progression to androgen-independent regulation of prostate-specific antigen gene in the $\mathrm{LNCaP}$ prostate tumour model. J Steroid Biochem Mol Biol 1996;58:139-146. [PubMed: 8809195]

49. Wright JL, et al. Intermittent androgen deprivation: clinical experience and practical applications. Urol Clin North Am 2006;33:167-179. [PubMed: 16631455]

50. Higano CS, et al. Intermittent androgen suppression with leuprolide and flutamide for prostate cancer: a pilot study. Urology 1996;48:800-804. [PubMed: 8911533]

51. Higano $\mathrm{C}$, et al. Bone mineral density in patients with prostate cancer without bone metastases treated with intermittent androgen suppression. Urology 2004;64:1182-1186. [PubMed: 15596194]

52. Miller K, et al. Randomised prospective study of intermittent versus continuous androgen suppression in advanced prostate cancer. J Clin Oncol 2007;25(18 Suppl):5015. [abstract \#5015].

53. Calais da Silva FM, et al. Five years survival and risk of death in a phase III study of intermittent monotherapy versus continuous combined androgen deprivation. J Clin Oncology 2008;26(15 Suppl):16034. [abstract \#16034].

54. Tunn U, et al. Intermittent is as effective as continuous androgen deprivation in patients with PSA relapse after radical prostatectomy. J Urol 2004;171(4 Suppl):384. [abstract \#1458]. [PubMed: 14665937]

55. Calais da Silva FM, et al. Phase III intermittent MAB vs continuous MAB. J Clin Oncol 2006;24(18 Suppl):4513. [abstract \#4513].

56. Malkowicz SB. The role of diethylstilbestrol in the treatment of prostate cancer. Urology 2001;58:108-113. [PubMed: 11502463]

57. Ricke WA, et al. Prostatic hormonal carcinogenesis is mediated by in situ estrogen production and estrogen receptor alpha signaling. FASEB J 2008;22:1512-1520. [PubMed: 18055862]

58. Pravettoni A, et al. Estrogen receptor beta (ERbeta) and inhibition of prostate cancer cell proliferation: studies on the possible mechanism of action in DU145 cells. Mol Cell Endocrinol 2007;263:46-54. [PubMed: 17023111]

59. Landström M, et al. Estrogen treatment postpones the castration-induced dedifferentiation of Dunning R3327-PAP prostatic adenocarcinoma. Prostate 1994;25:10-18. [PubMed: 8022707]

60. Kitahara S, et al. Effects of intravenous administration of high-dose diethylstilbestrol diphosphate on serum hormonal levels in patients with hormone-refractory prostate cancer. Endocrin J 1999;46:659_ 664.

61. Byar D, Corle DK. Hormone therapy for prostate cancer: results of the Veterans Administration Cooperative Urological Research Group studies. NCI Monogr 1988;7:165-170. [PubMed: 3050535]

62. Ockrim JL, et al. Transdermal estradiol improves bone density when used as single agent therapy for prostate cancer. J Urol 2004;172:2203-2207. [PubMed: 15538232]

63. Purnell JQ, et al. Effects of transdermal estrogen on levels of lipids, lipase activity, and inflammatory markers in men with prostate cancer. J Lipid Res 2006;47:349-355. [PubMed: 16299398]

64. Bland LB, et al. Phase II study of transdermal estradiol in androgen-independent prostate carcinoma. Cancer 2005;103:717-723. [PubMed: 15641029]

65. Kandola S, et al. Transdermal oestrogen therapy as a second-line hormonal intervention in prostate cancer: a bad experience. BJU Int 2007;99:53-55. [PubMed: 17227492] 
66. Sawyers, CL., et al. Characterization of a new anti-androgen MDV-3100 effective in preclinical models of hormone refractory prostate cancer. Presented at the ASCO 2007 Prostate Cancer Symposium; February 22-24; Orlando, FL, USA. 2007. [abstract \#48]

67. Scher HI, et al. Phase I/II study of MDV3100 in patients (pts) with progressive castration-resistant prostate cancer (CRPC). J Clin Oncol 2008;26(15 Suppl):5006. [abstract \#5006].

68. Reid AHM, et al. CYP17 inhibition as a hormonal strategy for prostate cancer. Nat Clin Pract Urol 2008;5:610-620. [PubMed: 18985049]

69. Danila, DC., et al. Preliminary phase II results of abiraterone acetate in patients with castrationresistant metastatic prostate cancer after failure of docetaxel-based chemotherapy. Presented at the ASCO 2008 Genitourinary Cancers Symposium; February 14-16; San Francisco, CA, USA. 2008. [abstract \#3]

70. Attard G, et al. Phase I clinical trial of a selective inhibitor of CYP17, abiraterone acetate, confirms that castration-resistant prostate cancer commonly remains hormone driven. J Clin Oncol 2008;26:4563-4571. [PubMed: 18645193]

71. Vasaitis T, et al. Androgen receptor inactivation contributes to antitumor efficacy of 17[alpha]hydroxylase/17,20-lyase inhibitor 3[beta]-hydroxy-17-(1H-benzimidazole-1-yl)androsta-5,16diene in prostate cancer. Mol Cancer Ther 2008;7:2348-2357. [PubMed: 18723482]

72. Mostaghel EA, Nelson PS. Intracrine androgen metabolism in prostate cancer progression: mechanisms of castration resistance and therapeutic implications. Best Pract Res Clin Endocrinol Metab 2008;22:243-258. [PubMed: 18471783]

73. GlaxoSmithKline (online 10 December 2008) NCT00470834: Prostate Cancer Study In Men Who Have Failed First-Line Androgen Deprivation Therapy. [accessed 12 December 2008]. [http://clinicaltrials.gov/ct2/show/NCT00470834] 


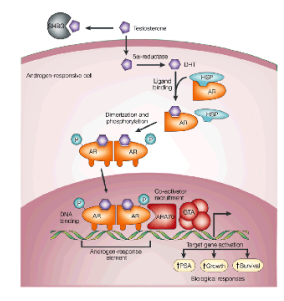

Figure 1. Androgen action

Testosterone circulates in the blood bound to albumin (not shown) and SHBG, and exchanges with free testosterone. Free testosterone enters prostate cells and is converted to DHT by the enzyme 5[alpha]-reductase. Binding of DHT to the AR induces dissociation from HSPs and receptor phosphorylation. The AR dimerizes and can bind to androgen-response elements in the promoter regions of target genes. Coactivators (such as ARA70) and corepressors (not shown) also bind the AR complex, facilitating or preventing, respectively, its interaction with the GTA. Activation (or repression) of target genes leads to biological responses including growth, survival and the production of PSA. Potential transcription-independent actions of androgens are not shown. Reproduced, with permission, from Feldman BJ and Feldman D (2001) Nat Rev Cancer 1: 34-45 (C) Macmillan Publishers Ltd. All rights reserved. Abbreviations: AR, androgen receptor; ARA70, androgen receptor associated protein 70; DHT, dihydrotestosterone; GTA, general transcription activation; HSP, heat-shock protein; SHBG, sex-hormone-binding globulin. 


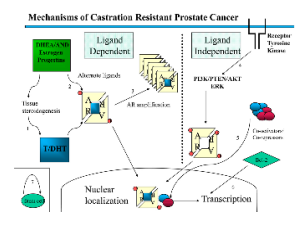

Figure 2. Mechanisms of castration resistance in prostate cancer

This Figure provides an overview of mechanisms demonstrated or hypothesized to be involved in the development of castration resistance in prostate cancer, divided into ligand-dependent and ligand-independent mechanisms. (1) Tissue and tumoral steroidogenesis contribute to synthesis of testosterone and DHT, and might lead to persistence of tissue-level androgen despite castration. (2) Mutations in the AR allow activation by alternate ligands or increased affinity for androgens. (3) Amplification increases AR abundance. (4) Ligand-independent activation of AR through ligand-independent modifications or cross-talk with other pathways, including phosphorylation of AR leading to hypersensitization and increased nuclear translocation. (5) Change in the balance of coactivators and corepressors augment AR activity. (6) Bypass pathways functioning independently of AR activity through upregulation of antiapoptotic molecules, such as Bcl-2. (7) Stem cells continuously produce both androgensensitive and castration-resistant clones. Abbreviations: AKT, akt serine/threonine kinase; AND, other androgenic steroidal precursors; AR, androgen receptor; DHEA, dihydroepiandrosterone; DHT, dihydrotestosterone; ERK, extracellular signal-regulated kinase; P, phosphorylated residues; PI3K, phosphoinositide 3-kinase; PTEN, phoshatase and tensin homolog. 


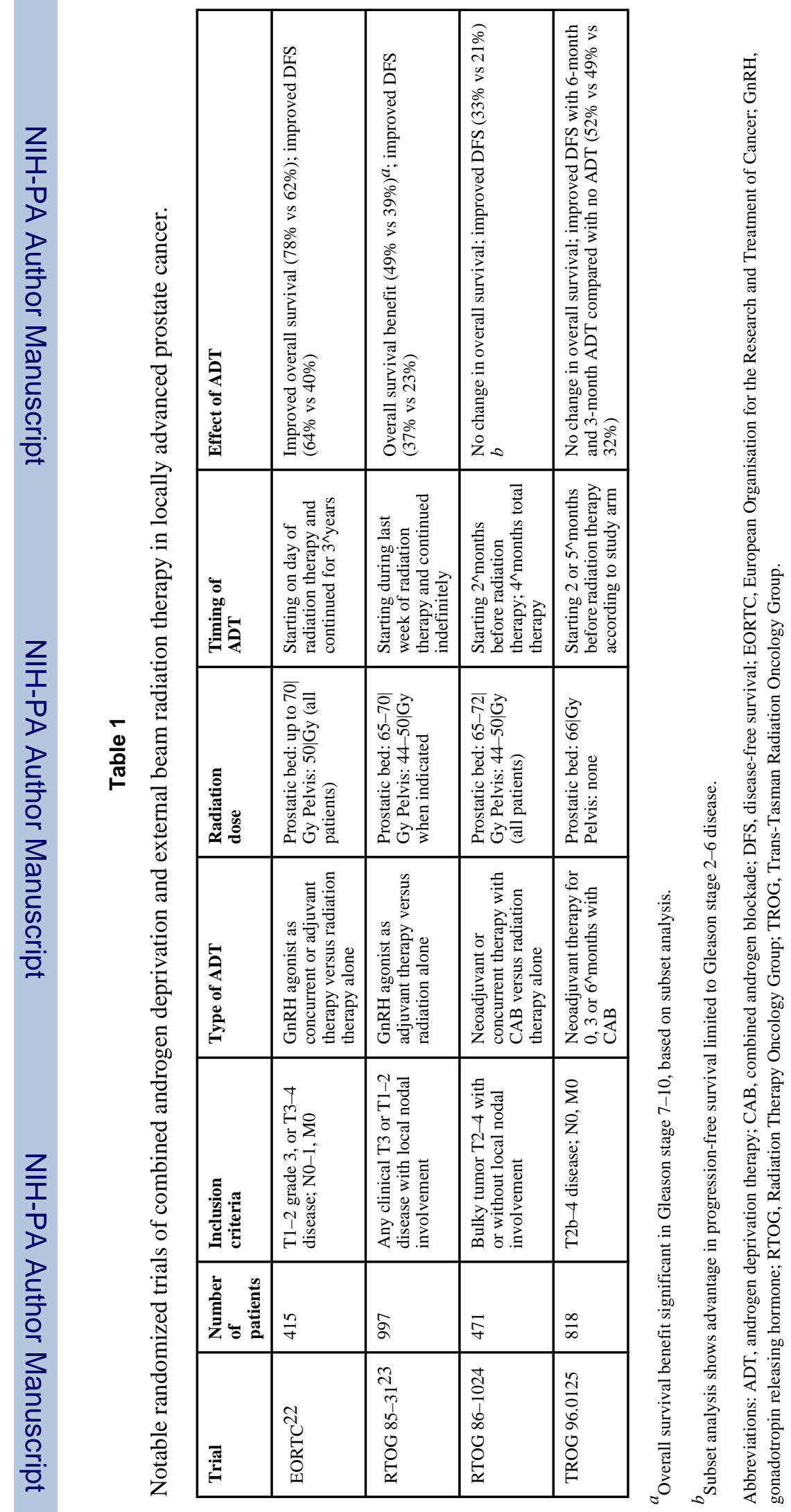

Nat Clin Pract Urol. Author manuscript; available in PMC 2010 November 15. 